International Mathematical Forum, 2, 2007, no. 35, 1737 - 1753

\title{
Existence of Maximal and Minimal Solutions of Differential Inclusion of Closed Interval-Valued Maps
}

\author{
A. M. Forouzanfar \\ Chamran University \\ Ahvaz, Iran
}

\begin{abstract}
Let $F(x)$ be a bounded closed interval-valued map. Then the differential inclusion, $D x(t) \in F(x(t)), x(0)=x_{0}, t \in[0, T]$ has maximal and minimal solutions, where $F$ is upper semi-continuous, $D$ is one of four Dini derivatives and $T>0$. Also this paper shows that general differential inclusion solvable and solutions depend continuously on the initial condition.
\end{abstract}

\section{Mathematics Subject Classifications: 35C, 35D}

Keywords: Differential inclusion, Upper (Lower) semi-continuous map

\section{INTRODUCTION}

Suppose $u$ and $v$ are bounded real valued functions. Let $F(x)=[v(x), u(x)]$ be closed interval-valued map. We consider differential inclusion,

$$
\text { (1) } D x(t) \in F(x(t)), x(0)=x_{0}, \quad t \in[0, T]
$$

where $D$ (throughout this paper) is one of four Dini derivatives as follow,

$D^{+} x(t)=\limsup _{s \rightarrow t_{+}}(x(s)-x(t)) /(s-t), \quad D^{-} x(t)=\limsup _{s \rightarrow t_{-}}(x(s)-x(t)) /(s-t)$

and $D_{+} x(t)$ and $D_{-} x(t)$ are defined as $D^{+} x(t)$ and $D^{-} x(t)$ respectively, when we use $\liminf$ instead of limsup, also $F: R \rightarrow 2^{R}$ is upper semi-continuous map that $2^{R}$ is the space of compact subsets of real numbers $R$, with the Vietoris topology. In this topology $R$ with usual topology, and a net $\left(A_{i}\right)$ converges to $A$ if and only if, given an open $U$ in $R$ with $A \subseteq U$ and a finite number of open sets $V_{1}, \cdots, V_{n}$ in $R$ with $A \cap V_{j} \neq \emptyset$ for $1 \leq j \leq n$, then 
eventually both $A_{i} \subseteq U$ and $A_{i} \cap V_{j} \neq \emptyset$ for $1 \leq j \leq n$. See [5] for more details.

A mapping $F: R \rightarrow 2^{R}$ is called upper semi-continuous (abbreviated to usc) if whenever $a_{i} \rightarrow a$ in $R$, then for each open set $U$ in $R$ with $F(a) \subseteq U$, eventually $F\left(a_{i}\right) \subseteq U$. If $F(x)$ is singlton, for each $x$, this means that $F$ is an ordinary continuous function. See [3].

In this paper we show that (1) has maximal (and minimal) solution so, it is solvable. To prove this we look at the maximal solution $\phi_{M}$ (and minimal solution $\phi_{m}$ ) of the differential inequalities,

$$
D x(t) \leq u(x(t)) \quad(\text { and } \quad D x(t) \geq v(x(t))), \quad x(0)=x_{0}, \quad t \in[0, T],
$$

where $u$ is bounded above upper semi-continuous, briefly usc (and $v$ is bounded below lower semi-continuous, briefly $l s c$ ) function that satisfies,

$$
\begin{gathered}
v\left(\phi_{M}(t)\right) \leq D \phi_{M}(t) \leq u\left(\phi_{M}(t)\right), \quad \phi_{M}(o)=x_{o}, \quad t \in[0, T], \\
\text { (and } \left.v\left(\phi_{m}(t)\right) \leq D \phi_{m}(t) \leq u\left(\phi_{m}(t)\right), \quad \phi_{m}(o)=x_{o}, \quad t \in[0, T]\right) .
\end{gathered}
$$

So, $\phi_{M}$ and $\phi_{m}$ are solutions of (1). In [1], he has shown that (2) has maximal (and minimal) solutions when $u$ and $v$ are continuous maps. In [4] has proved that if $v(x) \leq u(x)$ for all $x \in R$, then the set of solutions of $d x / d t=v(x(t)), t \in[o, T]$ is bounded by the maximal and minimal solutions of $d x / d t=u(x(t)), t \in[o, T]$, where $u(x)$ is continuous.

\section{PRELIMINARY}

The purpose of this section is to prove some results which we need later. By the definition of Dini derivatives,

1.1 COROLLARY. If $\phi:[a, b] \rightarrow R$ is a continuous map then,

(i) $D_{+} \phi \leq D^{+} \phi$ and $D_{-} \phi \leq D^{-} \phi$,

$$
\begin{aligned}
& D^{+}(-\phi)=-D_{+} \phi, \quad D_{+}(-\phi)=-D^{+} \phi, \quad D^{-}(-\phi)=-D_{-}(\phi) \\
& \text { and } D_{-}(-\phi)=-D^{-} \phi .
\end{aligned}
$$

1.2 LEMMA. Let $\phi:[a, b] \rightarrow R$ be a continuous map and $D^{+} \phi(t) \geq 0$ nearly every where (i.e. for all $t$, except countable subset) in $[a, b)$, then $\phi$ is increasing.

Proof. It is obviously enough to prove that $\phi(a) \leq \phi(b)$. First we show that if $\phi(a)>\phi(b)$ and $A=\left\{t \in[a, b) ; D^{+} \phi(t) \leq 0\right\}$, then $(\phi(b), \phi(a)) \subseteq$ $\phi(A)$. Let $x \in(\phi(b), \phi(a))$. By the intermediate value theorem, there is a greatest element $t_{x} \in(a, b)$ such that $\phi\left(t_{x}\right)=x$ and for $t_{x}<t \leq b$ we have 
$\phi(t)<x$ and so, $\phi(t)-\phi\left(t_{x}\right)<x-x=0$. Hence $D^{+} \phi\left(t_{x}\right) \leq 0$, i.e. $t_{x} \in A$. Therefore $x=\phi\left(t_{x}\right) \in \phi(A)$.

Now let $\epsilon>0$, and let $\psi(t)=\phi(t)+\epsilon t$. So, nearly every where in $[a, b), D^{+} \psi(t)$

$=D^{+} \phi(t)+\epsilon>0$, hence the set $\mathrm{t}$, where $D^{+} \psi(t) \leq 0$ is countable. Then the image of this set by $\psi$ is countable, and therefore contains no non-empty open interval. Now by the above argument we conclude that $\psi(a) \leq \psi(b)$. Since $\epsilon$ is arbitrary, this gives the result.

1.3 COROLLARY. If $\phi:[a, b] \rightarrow R$ is continuous and $D \phi(t) \geq 0$ nearly every where in $(a, b]$, then $\phi$ is increasing.

Proof. For $D=D^{-}$, this follows by applying (1.2) to the function $t \rightarrow \phi(a+b-t)$ on $[a, b]$. For $D=D_{+}$and $D=D_{-}$we use the facts that $D_{+}(-\phi)=-D^{+} \phi(t)$ and $D_{-}(-\phi)=-D^{-} \phi$.

1.4 LEMMA. Let $M \in R$ and $\phi:[a, b] \rightarrow R$ be continuous function such that $D \phi(t) \leq M$ nearly every where in $[a, b]$. Then $\phi(b)-\phi(a) \leq M(b-a)$.

Proof. $\quad$ Let us define $\psi(t)=\phi(a)-\phi(t)+M(t-a)$ for all $t \in[a, b]$. Obviously we have $D^{+} \psi(t)=M-D_{+} \phi(t), D^{-} \psi(t)=M-D_{-} \phi(t), D_{-} \psi(t)=$ $M-D^{-} \phi(t)$ and $D_{+} \psi(t)=M-D^{+} \phi(t)$. Then by (1.2) and (1.3), $\psi$ is increasing on $[a, b]$. But $\psi(a)=0$, therefor, $\psi(b) \geq 0$ and hence the inequality follows.

1.5 COROLLARY. Let $M \in R$ and $\phi:[a, b] \rightarrow R$ be a continuous map such that $D \phi(t) \geq M$ for nearly every where in $[a, b]$. Then $\phi(b)-\phi(a) \geq$ $M(b-a)$.

Proof. We prove it for $D=D^{+}$, in other the cases the proof will be similar. We have $D_{+}(-\phi)(t)=-D^{+} \phi(t) \leq-M$, so by $(1.4),(-\phi)(b)-$ $(-\phi)(a) \leq-M(b-a)$. Then the inequality follows.

The next result is a generalizing of $([1], 1.4 .5)$. He has proved it for continuous functions but we prove it for $l s c$ functions.

1.6 LEMMA. Let $\psi: R \rightarrow R$ be a lsc function and let $\phi:[a, b] \rightarrow R$ be a continuous map. If $D \phi(t) \geq \psi(t)$ nearly every where in $[a, b]$, then $D^{\prime} \phi(t) \geq$ $\psi(t)$ for all $t \in(a, b)$, where $D^{\prime}$ denotes a fixed one of the four Dini derivatives.

Proof. Let $\varepsilon>0$ be given, and $t \in(a, b)$. Since $\psi$ is $l s c$ at $t$, we can find $\delta>0$ such that $\psi(s) \geq \psi(t)-\varepsilon$ for all $s \in(t-\delta, t+\delta)$. Then $D \phi(s) \geq \psi(t)-\varepsilon$ nearly every where in $(t-\delta, t+\delta)$. Then by $(1.5), \phi(s)-\phi(t) \geq(\psi(t)-\varepsilon)(s-t)$, so we conclude that $D^{\prime} \phi(t) \geq \psi(t)-\varepsilon$. Since $\varepsilon$ is arbitrary, this gives the result.

1.7 COROLLARY. Let in (1.6), $\psi: R \rightarrow R$ be an usc function. If $D \phi(t) \leq \psi(t)$ nearly every where in $[a, b]$. Then $D^{\prime} \phi(t) \leq \psi(t)$ for all $t \in[a, b]$.

Proof. The proof is the same as the proof of (1.6). Only we use usc 
property instead of the lsc and obviously, the inequalities will be changed.

\section{DIFFERENTIAL INEQUALITIES}

Let $u: R \rightarrow R$ be an usc function, $k_{1}, k_{2} \in R$ and $T>0$. This section is devoted to existence of the maximal solution of the differential inequalities,

$$
D x(t) \leq u(x(t)), k_{1} \leq D x(t) \leq k_{2}, x(0)=x_{0}, \quad t \in[0, T]
$$

which we shall prove it is monotone. The topology on each set of functions will be the norm topology.

2.1 LEMMA. Let $T$ be a positive real number and $k_{1}, k_{2} \in R$. Then

$$
S=\left\{\phi:[0, T] \rightarrow R \mid \phi \text { continuous, } k_{1} \leq D \phi(t) \leq k_{2} \text { and } \phi(0)=x_{0}\right\}
$$

is closed compact equicontinuous set.

Proof. Let $\phi \in S$, so $D \phi(t) \leq \max \left\{\left|k_{1}\right|,\left|k_{2}\right|\right\}=k$. Then by $(1.4), \phi(t)-$ $\phi(s) \leq k(t-s)$ for all $0 \leq s \leq t \leq T$. Hence, obviously, $S$ is equicontinuous and bounded, so by the Arzela-Ascoli theorem $\operatorname{cls}(\mathrm{S})$ is compact. Suppose $\left(\phi_{n}\right)$ is a sequence in $S$ which converges to $\phi$. Since the topology is norm topology, so $\phi$ is continuous on $[0, T]$. Also for all $n, k_{1} \leq D \phi_{n}(t) \leq k_{2}$, then by (1.4) and (1.5), for $0 \leq s<t \leq T, k_{1}(t-s) \leq \phi_{n}(t)-\phi_{n}(s) \leq k_{2}(t-s)$. Hence $k_{1} \leq\left(\phi_{n}(t)-\phi_{n}(s)\right) /(t-s) \leq k_{2}$ for all $n$ and by taking the limit as $n \rightarrow \infty$, we obtain, $k_{1} \leq(\phi(t)-\phi(s)) /(t-s) \leq k_{2}$. If $D=D^{-}$we take lim sup or lim inf as $s \rightarrow t_{-}$, we have $k_{1} \leq D \phi(t) \leq k_{2}$. If $D=D^{+}$or $D=D_{+}$we consider $0 \leq t<s \leq T$, and as above, we have, $k_{1} \leq(\phi(s)-\phi(t)) /(s-t) \leq k_{2}$. By taking lim sup or liminf as $s \rightarrow t_{+}, k_{1} \leq D \phi(t) \leq k_{2}$ as desired.

Now we discuss "some compact subsets" of $S$ in (2.1).

2.2 LEMMA. Let $u: R \rightarrow R$ be a usc map and bounded above by $k_{2}$. Then the set $\Omega=\left\{\phi \in S \mid D \phi(t) \leq u(\phi(t)), \phi(0)=x_{0}\right\}$ is compact.

Proof. $\quad$ Since $S$ is compact, it is enough to show that $\Omega$ is closed. Let $\left(\phi_{n}\right)$ be a sequence in $\Omega$ such that $\phi_{n} \rightarrow \phi$. We have to show that $\phi \in \Omega$. Let $\varepsilon>0$ be given. By the usc property of $u$, there is a nbhd $V$ of $\phi\left(t_{0}\right)$ such that

$$
\text { (1) } u(y) \leq u\left(\phi\left(t_{0}\right)\right)+\varepsilon, \quad(y \in V) .
$$

Equicontinuity of $\Omega$ gives us that the map $(\psi, t) \rightarrow \psi(t): \Omega \times[0, T] \rightarrow R$ is continuous. Since $V$ is a nbhd of $\phi\left(t_{0}\right)$, then there are $\delta\left(t_{0}\right)>0$ and $N$ (not depending on $\delta\left(t_{0}\right)$ ) such that $\phi_{n}(t) \in V$ for all $\left|t-t_{0}\right| \leq \delta \& n \geq N$. Hence by (1), $u\left(\phi_{n}(t)\right) \leq u\left(\phi\left(t_{0}\right)\right)+\varepsilon$ for all $\left|t-t_{0}\right| \leq \delta \& n \geq N$. So by the definition of $\Omega, D \phi_{n}(t) \leq u\left(\phi\left(t_{0}\right)\right)+\varepsilon$ for $\left|t-t_{0}\right| \leq \delta$ and $n \geq N$. Then by (1.4), $\phi_{n}(t)-\phi_{n}\left(t_{0}\right)<\left(u\left(\phi\left(t_{0}\right)\right)+\varepsilon\right)\left(t-t_{0}\right)$ for all $t \in\left(t_{0}, t_{0}+\delta\right) \& n \geq N$. 
So, $\left(\phi_{n}(t)-\phi_{n}\left(t_{0}\right)\right) /\left(t-t_{0}\right) \leq u\left(\phi\left(t_{0}\right)\right)+\varepsilon$. By letting $n \rightarrow \infty$ we obtain, $\left(\phi(t)-\phi\left(t_{0}\right)\right) /\left(t-t_{0}\right) \leq u\left(\phi\left(t_{0}\right)\right)+\varepsilon$ for all $t \in\left(t_{0}, t_{0}+\delta\right)$. Also, similarly we have $\left(\phi\left(t_{0}\right)-\phi(t)\right)\left(t_{0}-t\right) \leq u\left(\phi\left(t_{0}\right)\right)+\varepsilon$ for all $t \in\left(t_{0}-\delta, t_{0}\right)$. Now by taking limit as desired, we conclude that, $D \phi\left(t_{0}\right) \leq u\left(\phi\left(t_{0}\right)\right)+\varepsilon$. Since $\varepsilon$ is arbitrary, $D \phi\left(t_{0}\right) \leq u\left(\phi\left(t_{0}\right)\right)$. By $(2.1), \mathrm{S}$ is closed, so $\phi \in S$ and it follows that $\phi \in \Omega$, as required.

In lemma (2.2) we can change us.continuity to ls.continuity in the following way.

2.3 COROLLARY. Let $v: R \rightarrow R$ be a lsc function which is bounded below by $k_{1}$. Then the set $\Omega^{\prime}=\left\{\phi \in S \mid D \phi(t) \geq v(\phi(t)), \phi(0)=x_{0}\right\}$ is compact.

Proof. The proof is as for lemma (2.2). We use ls.continuity instead of us.continuity and obviously, the inequalities are reversed.

In the following lemma we study the existence of a maximal member of $\Omega$.

2.4 THEOREM. $\quad$ The set $\Omega$ in (2.2) has a unique maximal element.

Proof. Let first, D be a fixed one of $D^{+}$or $D^{-}$. Suppose $\phi_{1}, \phi_{2} \in \Omega$. We observe that if $\phi_{1}(t)>\phi_{2}(t)$, then $D\left(\phi_{1} \vee \phi_{2}\right)(t)=D \phi_{1}(t)$. Also if $\phi_{1}(t)=$ $\phi_{2}(t), D\left(\phi_{1} \vee \phi_{2}\right)(t)=\max \left\{D \phi_{1}(t), D \phi_{2}(t)\right\}$, so it follows that $\phi_{1} \vee \phi_{2} \in \Omega$.

Now for each finite set $F=\left\{\phi_{1}, \cdots, \phi_{n}\right\}$ of elements of $\Omega$, we consider $U_{F}=\phi_{1} \vee \phi_{2} \vee \cdots \vee \phi_{n}$. Then, $\left\{U_{F}: F\right.$ any finite subset of $\left.\Omega\right\}$ is an increasing net which is indexed by the collection of all finite subsets of $\Omega$. Now let us take any convergent subnet $U_{F^{\prime}} \rightarrow \phi \in \Omega$ (this is allowed by the lemma (2.2)). We claim that , $\phi=\sup _{f \in \Omega} f$. Let $f \in \Omega$. Then if $f \in F$, so, $U_{f} \leq U_{F}$. Since $\left(U_{F^{\prime}}\right)$ is a subnet, there is $F_{0}^{\prime}$ such that whenever $F_{0}^{\prime} \subseteq F^{\prime}$, we have $F \subseteq F^{\prime}$, and so $U_{f} \leq U_{F} \leq U_{F^{\prime}}$. It follows that $f \leq \lim _{F^{\prime}} U_{F^{\prime}}=\phi$. Hence $\phi$ is an upper bound of $\Omega$. Assume $\phi^{\prime}$ is any upper bound for $\Omega$. Then $\phi^{\prime} \geq f_{i}, 1 \leq i \leq n$ for any $n$ elements of $\Omega$. We obtain that $\phi^{\prime} \geq f_{1} \vee f_{2} \cdots \vee f_{n}=U_{\left\{f_{1}, f_{2}, \cdots, f_{n}\right\}}$ and so, $\phi^{\prime} \geq U_{G}$ for all finite subset $G$ of $\Omega$. We deduce that $\phi^{\prime} \geq \lim U_{F^{\prime}}=\phi$ and thus $\sup \Omega=\phi$. Therefore $\phi$ is a maximal member of $\Omega$.

Let now, $D=D_{+}$or $D=D_{-}$. Since $D_{+} x(t) \leq D^{+} x(t)$, then each solution of, (1) $D^{+} x(t) \leq u(x(t)), x(0)=x_{0}$, is a solution of (2) $D_{+} x(t) \leq$ $u(x(t)), x(0)=x_{0}$. Suppose $\phi$ be a solution of (2). Since uo $\phi$ is usc, by (1.7), we have $D^{+} \phi(t) \leq u(\phi(t)), \phi(0)=x_{0}$. Thus the differential inequalities (1) and (2) have the same solutions. So (2) has unique maximal solution which is equal to the unique maximal solution of (1). Similarly for $D^{-} x(t) \leq u(x(t))$ and $D_{-} x(t) \leq u(x(t)), x(0)=x_{0}$. Therefore $\Omega$ has a maximal member. Since the maximal member of $\Omega$ is $\phi=\sup \Omega$, then it is unique.

2.5 LEMMA. Let $u: R \rightarrow R$ be usc map such that $u(x) \leq k_{2}$ for all $x \in R$, then the differential inequalities, (1) $D^{+} x(t) \leq u(x(t))$, (2) $D_{+} x(t) \leq u(x(t))$, (3) $D^{-} x(t) \leq u(x(t))$, (4) $D_{-} x(t) \leq u(x(t))$, with $k_{1} \leq D x(t) \leq k_{2}, x(0)=x_{0}$ 
and $t \in[0, T]$ have the same unique maximal solution.

Proof. We prove only (1) and (4) have the same unique maximal solution the proof for other cases will be similar. Let $\phi$ be a solution of (1). Since $u o \phi$ is $u s c$ so by $(1.7), D_{-} \phi(t) \leq u(\phi(t))$. Then every solution of $(1)$ is a solution of (4). Similarly, every solution of (4) is a solution of (1). Therefore (1) and (4) have the same maximal solution.

The parallel results to (2.4) and (2.5) hold when we use a lsc map $v$ instead of a usc map $u$. The proof will be similar.

2.6 COROLLARY. Suppose $v: R \rightarrow R$ is a lsc function with $v(x) \geq k_{1}$, for all $x \in R$. Then the set $\Omega$ has a unique minimal continuous solution.

Proof. We use lsc property instead of usc property in (2.4) and obviously the inequalities are reversed.

2.7 COROLLARY. Let $v: R \rightarrow R$ be the lsc function, then the differential inequalities $D^{+} x(t) \geq v(x(t)), D_{+} x(t) \geq v(x(t)), D^{-} x(t) \geq v(x(t)), D_{-} x(t) \geq$ $v(x(t))$, with $k_{2} \geq D x(t) \geq k_{1}, x(0)=x_{0}, t \in[0, T]$ have the same unique minimal solution.

Proof. The proof is exactly the same as the proof of (2.5).

In general, the minimal solution $\phi$ of the inequalities in (2.7) does not satisfy

$$
D \phi(t)=v(\phi(t)), \quad \phi(0)=x_{0}, \quad t \in[0, T] .
$$

Let $D=D^{+}$and $a, x_{0} \in R$ with $0 \leq x_{0}<a$. We define $v(x)=2$ for all $0 \leq x<a, v(x)=1 / 4$ for $x=a$ and $v(x)=1 / 2$ for all $x>a$. Obviously, $v$ is a $l s c$ real valued function. The minimal solution of $D^{+} x(t) \geq v(t(t)), x(0)=$ $x_{0}, \phi$ say, is a continuous function whose graph has slope 2 on $\left[0,\left(a-x_{0}\right) / 2\right]$ and slope $1 / 2$ on the rest (in fact $\phi$ is the unique continuous solution). But we have

$$
D^{+} \phi\left(\left(a-x_{0}\right) / 2\right)=1 / 2>v\left(\phi\left(\left(a-x_{0}\right) / 2\right)\right)=v(a)=1 / 4 .
$$

See the following figures. 


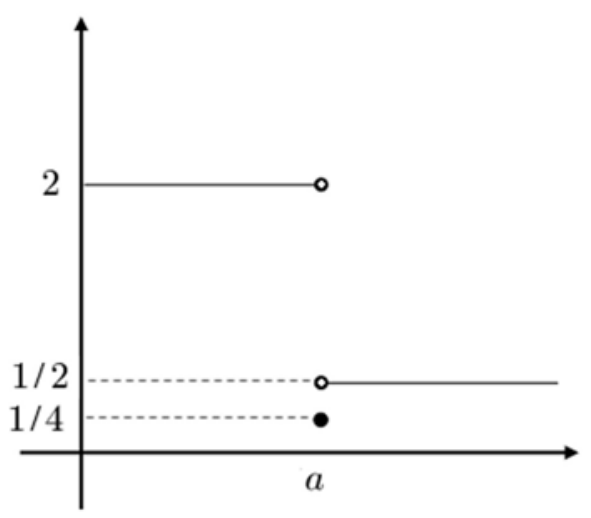

Figure 1: Graph of $v$.

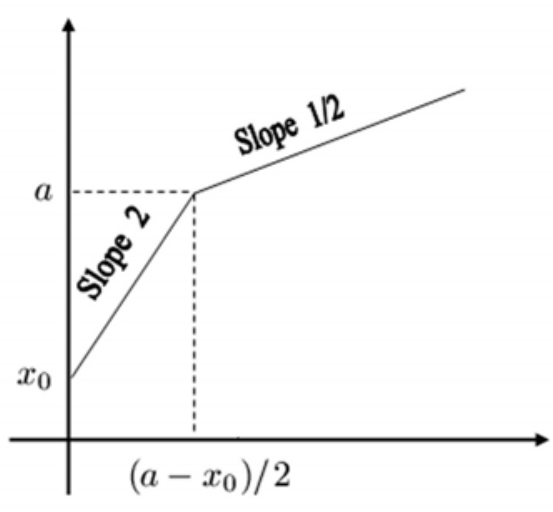

Figure 2: Graph of $\phi$.

Also, the same argument for the maximal solution of the inequalities (2.5) holds.

2.8 THEOREM. The maximal element of the set $\Omega$ in (2.2) is monotone.

Proof. Let $\phi$ be the maximal solution of the inequalities,

$$
k_{1} \leq D \phi(t) \leq k_{2}, D \phi(t) \leq u(\phi(t)), \phi(0)=x, t \in[0, T]
$$

and $\phi$ is not monotone. Then there are $0 \leq t_{1}<t_{2}<t_{3} \leq T$ such that,

$$
\phi\left(t_{1}\right)>\phi\left(t_{3}\right)>\phi\left(t_{2}\right) \quad\left(\text { or } \phi\left(t_{1}\right)<\phi\left(t_{3}\right)<\phi\left(t_{2}\right)\right) .
$$

Suppose, now, $M=\left(\phi\left(t_{2}\right)+\phi\left(t_{3}\right)\right) / 2$ so, $\phi\left(t_{2}\right)<M$ and $\phi\left(t_{3}\right)>M$. We define $s_{1}=\inf \left\{t \geq t_{1} ; \phi(t)=M\right\}$ and $s_{2}=\sup \left\{t \leq t_{3} ; \phi(t)=M\right\}$. Now there are two cases for $\phi(t)$ when $t>s_{2}$.

i) $\underline{\phi(t)>M \text { for } t>s_{2}}$.

We define the continuous map $\psi(t)$ to be equal to $\phi(t)$ on $\left[0, s_{1}\right]$ and $\phi(t+$ $\left.s_{2}-s_{1}\right)$ for all $t \geq s_{1}$. Obviously $D \psi(t) \leq u(\psi(t))$ for all $t \geq 0$, but, $\psi\left(t_{2}\right)=$ $\phi\left(t_{2}+s_{2}-s_{1}\right)>M>\phi\left(t_{2}\right)$, because $t_{2}+s_{2}-s_{1}>s_{2}$. Thus, this is a contradiction to maximality of $\phi$.

ii) $\phi(s)=M$ for some $s>s_{2}$.

Assume $s_{0}=\inf \left\{s>t_{3} ; \phi(s)=M\right\}$. Then, $s_{0}>s_{2}, \phi(t) \geq M$ for $t \in\left[s_{2}, s_{0}\right]$. Put $l=s_{0}-s_{2}$. Define $\psi(t)$ to be equal to $\phi(t)$ on $\left[0, s_{1}\right]$ and to be periodic of period $l$ from $s_{1}$. i.e. $\psi(t)=\phi(t)$ for $t \in\left[0, s_{1}\right], \psi(t)=$ $\phi\left(t+s_{2}-s_{1}-k l\right)$ for all $t$, with $s_{1}+k l \leq t \leq s_{1}+(k+1) l \leq T, k=0,1,2, \cdots, N$, for some $N$. Since $\phi\left(t+s_{2}-s_{1}\right)>M$ for $s_{1} \leq t \leq s_{1}+l$ then $\phi(s) \geq M$ for all $s \geq s_{1}$. Let $s_{1} \leq t \leq T$, then,

$$
D \psi(t)=D \phi\left(t+s_{2}-s_{1}-k l\right) \leq u\left(\phi\left(t+s_{2}-s_{1}-k l\right)\right)=u(\psi(t))
$$


for some $k=0, \cdots, N$; so $\psi$ is a solution of the inequality. But by the definition of $\psi, \psi\left(t_{2}\right)=\phi\left(t_{2}+s_{2}-s_{1}-k l\right) \geq M>\phi\left(t_{2}\right)$ and this is a contradiction to maximality of $\phi$.

In the case of $\phi\left(t_{1}\right)<\phi\left(t_{3}\right)<\phi\left(t_{2}\right)$ the proof will be parallel. Hence $\phi$ is monotone.

2.9 COROLLARY. Let $v: R \rightarrow R$ be lsc map with $v(x) \geq k_{1}$ for all $x \in R$, and $k_{1}, k_{2} \in R$. Then the minimal continuous solution $\phi$ of the inequalities, $k_{1} \leq D \phi(t) \leq k_{2}, D \phi(t) \geq v(\phi(t)), \phi(0)=x_{0}, t \in[0, T]$ is monotone.

Proof. The proof will be the same as the proof of (2.8), we use only the minimality of the solution instead of the maximality of the solution.

\section{DIFFERENTIAL INCLUSION}

Assume $u$ and $v$ are real functions and let $F(x)=[v(x), u(x)]$ be bounded closed interval-valued map, then there are $k_{1}, k_{2} \in R$, such that $F(x) \subseteq\left[k_{1}, k_{2}\right]$, for all $x$. In this section we show that maximal and minimal solutions of the differential inclusion

$$
D x(t) \in F(x(t)), \quad x(0)=x_{0}, \quad t \in[0, T],
$$

are exist (so, it is solvable); where $F$ is usc map. The maximal solution is equal to the maximal solution of $D x(t) \leq u(x(t))$ and the minimal solution is equal to the minimal solution of $D x(t) \geq v(x(t))$ that $x(0)=x_{0}$ and $t \in[0, T]$.

In the following lemma, we study the relation between closed intervalvalued map $F$ and real functions $u$ and $v$.

3.1 LEMMA. Let $F(x)=[v(x), u(x)]$ be a set valued map as above. Then $u$ and $v$ are usc and lsc as real functions respectively if and only if $F$ is a usc set-valued map.

Proof. Let $u$ and $v$ be $u s c$ and $l s c$ respectively and $\varepsilon>0$ be given. Then, for $x_{0} \in R$, by us.continuity and ls.continuity there are nbhds $N_{1}\left(x_{0}\right)$ and $N_{2}\left(x_{0}\right)$ of $x_{0}$ such that, $u(x) \leq u\left(x_{0}\right)+\varepsilon / 2\left(x \in N_{1}\left(x_{0}\right)\right), v(x) \geq v\left(x_{0}\right)-$ $\varepsilon / 2\left(x \in N_{2}\left(x_{0}\right)\right)$. Hence, for all $x \in N_{1}\left(x_{0}\right) \cap N_{2}\left(x_{0}\right),[v(x), u(x)] \subseteq\left(v\left(x_{0}\right)-\right.$ $\left.\varepsilon, u\left(x_{0}\right)+\varepsilon\right)$. Therefore $F$ is usc.

Conversely, let $\left(v\left(x_{0}\right)-\varepsilon, u\left(x_{0}\right)+\varepsilon\right)$ be an open nbhd of $F\left(x_{0}\right)=\left[v\left(x_{0}\right), u\left(x_{0}\right)\right]$, so by us.continuity of $F$, there is a nbhd $N\left(x_{0}\right)$ of $x_{0}$ such that $F(x) \subset$ $\left(v\left(x_{0}\right)-\varepsilon, u\left(x_{0}\right)+\varepsilon\right)$ for all $x \in N\left(x_{0}\right)$. Then $v(x)>v\left(x_{0}\right)-\varepsilon, u(x)<u\left(x_{0}\right)+\varepsilon$ for all $x \in N\left(x_{0}\right)$ as required.

Now we combine the lemmas (2.2), (2.3), (2.5), (2.7) and (2.9).

3.2 LEMMA. Let $F(x)=[v(x), u(x)]$ be bounded usc closed intervalvalued map. Then the set,

$$
\Lambda=\left\{\phi:[0, T] \rightarrow R \mid \phi \text { is continuous, } D \phi(t) \in F(\phi(t)), \phi(0)=x_{0}\right\},
$$


is compact.

Proof. Since $F$ is bounded, then there are $k_{1}, k_{2} \in R$, such that $F(x)=$ $[v(x), u(x)] \subseteq\left[k_{1}, k_{2}\right]$, for all $x$. Then $\Lambda=\Omega \cap \Omega^{\prime}$, and since $\Omega$ and $\Omega^{\prime}$ are compact, then, $\Lambda$ is compact.

3.3 LEMMA. Let $F(x)=[v(x), u(x)]$ be bounded usc closed intervalvalued map and $\phi_{M}$ be the maximal element of $\Omega$. Then $D \phi_{M}(t) \geq v\left(\phi_{M}(t)\right), \phi_{M}(0)=$ $x_{0}$ for all $t \in[0, T]$. Hence $D \phi_{M}(t) \in F\left(\phi_{M}(t)\right)$, for all $t \in[0, T]$.

Proof. Assume $D=D^{+}$or $D=D_{+}$and $t \in[0, T]$. Let $\varepsilon>0$ be given. By ls.continuity of $v o \phi_{M}$ at $t_{0}$ there is $\delta>0$ such that, $u\left(\phi_{M}(t)\right) \geq$ $v\left(\phi_{M}(t)\right)>v\left(\phi_{M}\left(t_{0}\right)\right)-\varepsilon$ for all $t \in\left(t_{0}-\delta, t_{0}+\delta\right)$. We define now, $\psi$ equals to $\phi_{M}$ on $\left[0, t_{0}\right]$ and equals to $\left(t-t_{0}\right)\left(v\left(\phi_{M}\left(t_{0}\right)\right)-\varepsilon\right)+\phi_{M}\left(t_{0}\right)$ on $\left[t_{0}, t_{0}+\delta\right]$ and whose graph on the rest of $[0, T]$ is a line segment of slope $k_{1}$. Clearly, $D \psi(t)=k_{1} \leq u(\psi(t))$ for all $T>t \geq t_{0}+\delta$, because we assumed $F$ is bounded so, $k_{1} \leq v(x) \leq u(x)$ for all $x$. Then we conclude that $D \psi(t) \leq u(\psi(t))$ for all $t \in[0 . T]$, so by maximality of $\phi_{M}$, we have $\phi_{M}(t) \geq \psi(t)$ for all $t \in[0, T]$. Since $\phi_{M}\left(t_{0}\right)=\psi\left(t_{0}\right),\left(\phi_{M}(t)-\phi_{M}\left(t_{0}\right)\right) /\left(t-t_{0}\right) \geq\left(\psi(t)-\psi\left(t_{0}\right)\right) /\left(t-t_{0}\right)$ for all $t \in\left(t_{0}, t_{0}+\delta\right)$. But the right hand side of the inequality is the slope of $\psi$ on $\left[t_{0}, t_{0}+\delta\right]$, therefore $D \phi_{M}\left(t_{0}\right) \geq v\left(\phi_{M}\left(t_{0}\right)\right)-\varepsilon$. Since $\varepsilon$ is arbitrary. $D \phi_{M}\left(t_{0}\right) \geq v\left(\phi_{M}\left(t_{0}\right)\right)$. For $D=D^{-}$or $D=D_{-}$, we define $\psi$ equals to $\phi_{M}$ on $\left[0, t_{0}-\delta\right]$, and equals to $\left(t-t_{0}\right)\left(v\left(\phi_{M}\left(t_{0}\right)\right)-\varepsilon\right)+\phi_{M}\left(t_{0}\right)$ on $\left[t_{0}-\delta, t_{0}\right]$ and whose graph on the rest of $[0, T]$ is a line segment of slope $k_{1}$. As in the above argument, $D \phi_{M}\left(t_{0}\right) \geq v\left(\phi_{M}\left(t_{0}\right)\right)$. Since $t_{0}$ is arbitrary in $[0, T]$, then $D \phi_{M}(t) \in\left[v\left(\phi_{M}(t)\right), u\left(\phi_{M}(t)\right)\right]=F\left(\phi_{M}(t)\right)$ for all $t \in[0, T]$.

3.4 COROLLARY. Let $F(x)=[v(x), u(x)]$ be bounded usc set-valued map and $\phi_{m}$ be the minimal element of $\Omega$. Then $D \phi_{m}(t) \leq u\left(\phi_{m}(t)\right), \phi_{m}(0)=x_{0}$ for all $t \in[0, T]$. Hence $D \phi_{m}(t) \in F\left(\phi_{m}(t)\right)$ for all $t \in[0, T]$.

Proof. $\quad$ Proof will be parallel to (3.3). We use lsc property instead of usc property and obviously, the inequalities are reversed.

3.5 THEOREM. Let $F(x)=[v(x), u(x)]$ be a usc bounded closed intervalvalued map. Then, $D x(t) \in F(x(t)), x(0)=x_{0}, t \in[0, T]$ has maximal and minimal solutions. the maximal solution is equal to the maximal solution of $D x(t) \leq u(x(t)), x(0)=x, t \in[0, T]$, and the minimal solution is equal to the minimal solution of $D x(t) \geq v(x), x(0)=x_{0}, t \in[0, T]$.

Proof. Let $\phi_{m}$ be the minimal solution of (1) $D x(t) \geq v(x(t)), x(0)=$ $x_{0}, t \in[0, T]$. By (3.4), $\phi_{m}$ is a solution of $(2) D x(t) \in F(x(t)), x(0)=x_{0}, t \in$ $[0, T]$. And obviously if $\psi$ is a solution of $(2)$, then $D \psi(t) \geq v(\psi(t))$. So, by minimality of $\phi_{m}$ we have $\psi \geq \phi_{m}$. Therefore $\phi_{m}$ is a minimal solution of (2). For maximal solution is parallel. Converse by (3.3) and (3.4) trivial.

3.6 COROLlARY. Let $f:[0, T] \rightarrow R$ be a continuous function. Then 
$D x(t)=f(x(t)), x(0)=x_{0}$ is solvable.

Proof. Since $f$ is continuous on $[0, T]$, then $f$ is bounded. Now, by (3.5) the assertion follows.

\section{GENERAL DIFFERENTIAL INCLUSION}

In this section we define right and left (set-valued) derivatives of a continuous function in general. Then we prove the existence of the right and the left derivatives. Also we show that the sets of the right and left derivatives as set-value maps are not usc not lsc.

4.1 Definition. $\quad$ Let $x:[0, T] \rightarrow R$ be a continuous map. We define $\left(S_{D} x\right)(t)$, set-valued derivative of $x$ at $t$, as follows,

$$
\begin{gathered}
\left(S_{D}^{+} x\right)(t)=\left\{\text { all limit points of }(x(s)-x(t)) /(s-t) \text { as } s \rightarrow t_{+}\right\}, \\
\left(S_{D}^{-} x\right)(t)=\left\{\text { all limit points of }(x(s)-x(t)) /(s-t) \text { as } s \rightarrow t_{-}\right\}, \\
\left(S_{D} x\right)(t)=\left(S_{D}^{+} x\right)(t) \cup\left(S_{D}^{-} x\right)(t) .
\end{gathered}
$$

We call $\left(S_{D}^{+} x\right)(t)$ and $\left(S_{D}^{-} x\right)(t)$, right and left set-valued derivatives of $x$ at $t$ respectively. Obviously, $\left(S_{D}^{+} x\right)(t)$ on $(0, T]$ and $\left(S_{D}^{-} x\right)(t)$ on $[0, T)$ are defined.

4.2 THEOREM. Let $F(x)=[v(x), u(x)]$ be a usc closed intervalvalued which $k_{1} \leq F(x) \leq k_{2}$ for all $x \in R$. Then $\left(S_{D} x\right)(t) \in F(x(t)), t \in$ $[0, T], x(0)=x_{0}$ is solvable.

This theorem can be obtained by the theorem (3.5). Because, if $\phi_{m}$ is the minimal solution of $D x(t) \in F(x(t)), x(0)=x_{0}, t \in[0, T]$, we conclude that,

$$
u\left(\phi_{m}(t)\right) \geq D^{+} \phi_{m}(t) \geq D_{+} \phi_{m}(t) \geq v\left(\phi_{m}(t)\right) .
$$

Hence by definition of $D^{+}$and $D_{+}$,

$$
\left(S_{D}^{+} \phi_{m}\right)(t) \subseteq\left[v\left(\phi_{m}(t)\right), u\left(\phi_{m}(t)\right)\right], t \in[0, T] .
$$

Similarly, $\left(S_{D}^{-} \phi_{m}\right)(t) \subseteq F\left(\phi_{m}(t)\right)$, then $\left(S_{D} \phi_{m}\right)(t) \subseteq F\left(\phi_{m}(t)\right)$ for all $t \in[0, T]$.

The following proof is just an alternative way of obtaining the results.

Proof. $\quad$ Let $T$ be a large positive real number. Let $\phi_{1}:[0, T] \rightarrow R$ be any continuous function with $\phi_{1}(0)=x_{0}$, for which its graph consists of a finite number of straight line segments with vertices at the points of any partition of $[0, T]$,

$$
P_{1}=\left\{0=t_{0}, t_{1}, \cdots, t_{N}=T\right\}
$$

Construct $\phi_{1}$ inductively by,

$$
\phi_{1}(0)=x_{0}, \phi_{1}(t)=m_{i}\left(t-t_{i-1}\right)+\phi_{1}\left(t_{i-1}\right) \quad\left(t_{i-1} \leq t \leq t_{i}\right)
$$


where $m_{i}$ is arbitrarily chosen in $F\left(\phi_{1}\left(t_{i-1}\right)\right)$ for $(1 \leq i \leq N)$. Suppose now, $0 \leq s \leq t \leq T$, so there exist $t_{i}, \cdots, t_{j}$ such that $t_{i} \leq s<t_{i+1}<\cdots<t \leq t_{j}$. Since $F$ is bounded by $k=\max \left\{\left|k_{1}\right|,\left|k_{2}\right|\right\}$, then we obtain that,

$\left|\phi_{1}(t)-\phi_{1}(s)\right| \leq\left|m_{i+1}\right|\left(t_{i+1}-s\right)+\left|m_{i+2}\right|\left(t_{i+2}-t_{i+1}\right)+\cdots+\left|m_{j}\right|\left(t-t_{j-1}\right) \leq k(t-s)$.

Thus $\phi_{1}$ is bounded and continuous. Assume $\phi_{2}$ is made on $[0, T]$ by a similar method as $\phi_{1}$. Its graph consists of straight line segments with successive vertices at points of the partition,

$$
P_{2}=\left\{0, t_{0},\left(t_{0}+t_{1}\right) / 2, t_{1}, \cdots, t_{N-1},\left(t_{N-1}+t_{N}\right) / 2, t_{N}\right\} .
$$

Let us continue this process, then we obtain a continuous function $\phi_{2^{n}}$ with its graph consisting of straight line segments with successive vertices at points of $P_{2^{n}}=\left\{0, t_{0}^{\prime}, t_{1}^{\prime}, \cdots, t_{p}^{\prime}\right\}$ in which each subinterval is made by dividing each subinterval of $P_{2^{n-1}}$ at its mid-point. Since $F$ is bounded by $k$, for $0 \leq s \leq$ $t \leq T$ we have,

$$
\left|\phi_{2^{n}}(t)-\phi_{2^{n}}(s)\right| \leq k(t-s),
$$

so $\phi_{2^{n}}$ is bounded and continuous.

The sequence $\left(\phi_{2^{n}}\right)_{n=0}$ which is established in this method is bounded and obviously eqicontinuous. By the Arzela-Ascoli theorem it has a convergent subsequence $\left(\phi_{i\left(2^{n}\right)}\right)$ which uniformly converges to $\phi$ say. Hence $\phi$ is continuous.

Let $s \in[0, T]$ and $U$ be a closed nbhd of $F(\phi(s))$. By us.continuity of $F$ there is a nbhd $W$ of $\phi(s)$ such that $F(y) \subset U$ for all $y \in W$. By equicontinuity there are $M$ and $\delta>0$ such that $\phi_{i\left(2^{n}\right)}(t) \in W(\phi(s))$ for $i\left(2^{n}\right) \geq M$ and $t \in(s-\delta, s+\delta)(\delta$ not depending on $M)$. So we deduce that,

$$
F\left(\phi_{i\left(2^{n}\right)}(t)\right) \subseteq U \text { for } i\left(2^{n}\right) \geq M \text { and all } t \in(s-\delta, s+\delta) .
$$

Assume now, $i\left(2^{n}\right) \geq M, t \in(s-\delta, s+\delta)$. Since $D^{+} \phi_{i\left(2^{n}\right)}(t)$ is the slope of one segment of the graph of $\phi_{i\left(2^{n}\right)}$ in $(s-\delta, s+\delta)$, thus there exists $t_{j} \in(s-\delta, s+\delta)$ such that

$$
D^{+} \phi_{i\left(2^{n}\right)}(t) \in F\left(\phi_{i\left(2^{n}\right)}\left(t_{j}\right)\right) \subseteq U(F(\phi(s))) .
$$

Hence by (1.4) and (1.5) we conclude that

$$
\left(\phi_{i\left(2^{n}\right)}(t)-\phi_{i\left(2^{n}\right)}(s)\right) /(t-s) \in U(F(\phi(s)))
$$

for all $t \in(s-\delta, s+\delta), t \neq s$ and $i\left(2^{n}\right) \geq M$. Now, let $i\left(2^{n}\right) \rightarrow \infty$ then $(\phi(t)-\phi(s)) /(t-s) \in U(F(\phi(s)))$ for all $t \in(s-\delta, s+\delta), t \neq s$ because $U$ is closed. Then $\left(S_{D} \phi\right)(s) \subset U(F(\phi(s)))$. Since $U$ is arbitrary closed nbhd of $F(\phi(s))$ then we conclude that $\left(S_{D} \phi\right)(s) \subseteq F(\phi(s))$.

If $s=0$ or $s=T$ we consider the right differential $\left(S_{D}^{+} \phi\right)(0)$ or the left differential $\left(S_{D}^{-} \phi\right)(T)$ of $\phi$ at 0 or T. 
4.3 Example. Let $\phi$ be a continuous map from $[0, T]$ to $R$. Then $t \rightarrow S_{D}^{+} \phi(t)$ and $t \rightarrow S_{D}^{-} \phi(t)$ are not necessarily usc or $l s c$, nor is $t \rightarrow S_{D} \phi(t)$.

Proof. Let us consider coordinate axes. Choose the interval $[0, T]$ on the $x$-axis and on the y-axis too. Now we draw two lines through the origin, $\Delta$ and $\Delta^{\prime}$ with slopes $m$ and $m^{\prime}$ respectively with $0<m<m^{\prime}$. Let $0<a<m, m^{\prime}<b<\infty$. Let $\phi$ be the continuous function with its graph consisting of the straight line segments, $\Delta_{1}, \Delta_{2}, \Delta_{3}, \cdots$ as follows: $\Delta_{1}$ with the slope $a$ passing through the point $(T, T)$; meets $\Delta^{\prime}$ at $A_{1}=\left(t_{1}, x_{1}\right)$ (since slope of $\Delta_{1}$ is less than $m$ ). $\Delta_{2}$ with slope $b$ passing through $A_{1}$ and intersects $\Delta$ at $A_{2}=\left(t_{2}, x_{2}\right)$ (since slope of $\Delta_{2}$ is bigger than $m^{\prime}$ ). By continuing this process we will find continuous map $\phi$ whose graph consists of $\left\{\Delta_{i}: i=1,2, \cdots\right\}$. For each number $\alpha$ with $m \leq \alpha \leq m^{\prime}$, let the points of intersection of $y=\alpha x$ with $y=\phi(x)$ be $\left(s_{n}, \phi\left(s_{n}\right)\right)$ with $s_{n} \searrow 0$. Therefore

$$
\left(\phi\left(s_{n}\right)-\phi(0)\right) /\left(s_{n}-0\right)=\left(\phi\left(s_{n}\right)\right) /\left(s_{n}\right) \rightarrow \alpha .
$$

So, we have $S_{D}^{+} \phi(0)=\left[m, m^{\prime}\right]$. Now, let $\left(r_{n}, \phi\left(r_{n}\right)\right)$ be the points of intersection of $y=m x$ (i.e. $\Delta^{\prime}$ with $\left.y=\phi(x)\right)$ where $r_{n} \searrow 0$. Then $\left(S_{D}^{+} \phi\right)\left(r_{n}\right)=\{b\}$ and $\left(S_{D}^{-} \phi\right)\left(r_{n}\right)=\{a\}$ for all $n$. The assertion now follows. 


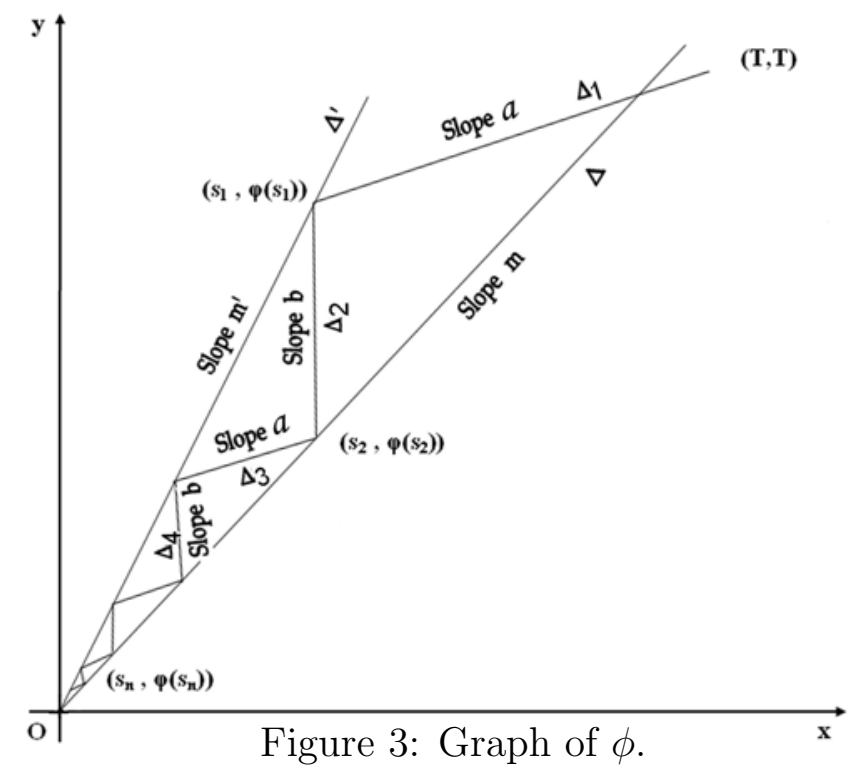

\section{INITIAL CONDITION}

Let $f: R \rightarrow R$ be a given function and $x^{\prime}=f(x)$ be an ordinary differential equation $\left(x^{\prime}(t)=d x / d t\right)$. An initial condition for a solution $x: R^{+} \rightarrow R$ is a condition of the from $x\left(t_{0}\right)=x_{0}$ where $t_{0} \in R^{+}$and $x_{0} \in R$. For simplicity usually we take $t_{0}=0$, so $x(0)=x_{0}$.

Suppose $\left(x_{n}\right)$ is a sequence in $R$ such that $x_{n} \rightarrow x_{0}$. Let $\phi_{n}$ be a solution of $x^{\prime}=f(x)$, with initial condition $x(0)=x_{n}$, for all $n$ and $\phi$ be a solution of $x^{\prime}=f(x)$ with $x(0)=x_{0}$. Can we say, $\phi_{n} \rightarrow \phi$ as $n \rightarrow \infty$ with respect to the sup norm? The answer is no, except under some conditions. For example let $f(x)=3 x^{2 / 3}$ with $x(0)=x_{n}$, such that $x_{n} \rightarrow 0$. Then $\phi_{n}(t)=\left(t+\sqrt[3]{x_{n}}\right)^{3}$ is a solution of the differential equation for all $n$. Also the map $\psi(t)=0$ for all $t$, is a solution of the differential equation with initial condition $x(0)=0$. But obviously, $\left(\phi_{n}\right)$ doesn't tend to $\psi$ with respect to sup norm.

Let $v: R \rightarrow R$ be an lsc function with $k_{1} \leq v(x) \leq k_{2}$ for all $x \in R$. Consider the differential inequality $D x(t) \geq v(x(t))$ with $x(0)=x_{n}, x_{n} \in R$ and $t \in[0, T]$. Suppose $\phi_{n}$ to be the minimal solution of the differential inequality. In this section we investigate the question whether, if $x_{n} \rightarrow x_{0}$, then $\phi_{n} \rightarrow \phi$ uniformly on $[0, T]$, where $\phi$ is the minimal solution of $D \phi(t) \geq$ $v(\phi(t)), \phi(0)=x_{0}$. Also we have shown that if $k_{1}>0$ or $k_{2}<0$, and $\phi(t, x)$ is a minimal solution of $D x(t) \geq v(x(t)), x(0)=x$ for $t \in[0, T]$. Then $(t, x) \rightarrow \phi(t, x)$ is continuous.

5.1 LEMMA. Let $v: R \rightarrow R$ be an lsc map. Suppose $\phi_{n} \rightarrow \phi$ uniformly, that $D \phi_{n}(t) \geq v\left(\phi_{n}(t)\right)$ for $t \in[0, T], \phi_{n}(0)=x_{n}$ for all $n$ and $x_{n} \rightarrow x_{0}$. Then $D \phi(t) \geq v(\phi(t))$ for $t \in[0, T], \phi(0)=x_{0}$. 
Proof. Since $\phi_{n} \rightarrow \phi$, with respect to the sup norm and $F=\left\{\phi_{n} ; n=\right.$ $1,2, \cdots\} \cup\{\phi\}$ is compact, then the set $F$ is equicontinuous. Let $\varepsilon>0$ be given and $t \in R$. By the lsc property of $v$, there exists a nbhd $W$ of $\phi(t)$ such that $v(y) \geq v(\phi(t))-\varepsilon$, for all $y \in W(\phi(t))$. By equicontinuity there are $\delta>0$ and $N$ (each of them not depending on the other) such that, $D \phi_{n}(s) \geq$ $v\left(\phi_{n}(s)\right) \geq v(\phi(t))-\varepsilon$ for all $s \in(t-\delta, t+\delta)$ and $n \geq N$. Hence by (1.4), $\phi_{n}(s)-\phi_{n}(t) \geq(v(\phi(t))-\varepsilon)(s-t), s \in(t, t+\delta)$ and also, $\phi_{n}(t)-\phi_{n}(s) \geq$ $(v(\phi(t))-\varepsilon)(t-s), s \in(t-\delta, t)$. By taking the limit as $n \rightarrow \infty$, we have $\phi(s)-\phi(t) \geq(v(\phi(t))-\varepsilon)(s-t), s \in(t, t+\delta), \phi(t)-\phi(s) \geq(v(\phi(t))-$ $\varepsilon(t-s), s \in(t-\delta, t)$. Then we conclude that $D \phi(t)>v(\phi(t))-\varepsilon$. Since $\varepsilon$ is arbitrary, $D \phi(t) \geq v(\phi(t))$ and $\phi(0)=x_{0}$ because $\phi_{n}(0) \rightarrow \phi(0)$.

5.2 COROLLARY. Let $u: R \rightarrow R$ be usc map. Suppose $\phi_{n} \rightarrow \phi$ uniformly on $[0, T]$ that $D \phi_{n}(t) \leq u\left(\phi_{n}(t)\right)$ for $t \in[0, T], \phi_{n}(0)=x_{n}$ for all $n$ and $x_{n} \rightarrow x_{0}$. Then, $D \phi(t) \leq u(\phi(t)), \phi(0)=x_{0}$.

Proof. The proof is exactly the same as (5.1). Just we use usc instead of $l s c$ and reverse the inequalities.

5.3 COROLLARY. Let $F(x)=[v(x), u(x)]$ be usc closed interval-valued map. Assume $\phi_{n} \rightarrow \phi$ uniformly on $[0, T]$, that $D \phi_{n}(t) \in F\left(\phi_{n}(t)\right)$ for $t \in$ $[0, T], \phi_{n}(0)=x_{n}$ for all $n$ and $x_{n} \rightarrow x_{0}$. Then $D \phi(t) \in F(\phi(t)), \phi(0)=x_{0}$ for all $t \in[0, T]$.

Proof. The proof by (5.1) and (5.2) is obvious.

5.4 LEMMA. Let $v: R \rightarrow R$ be lsc map such that $0<k_{1} \leq v(x) \leq k_{2}$ for all $x$. Let $\phi_{n}$ be the minimal solution of $D x(t) \geq v(x(t))$ for $t \in[0, T]$, $x(0)=x_{n}$ for all $n$, where $\left(x_{n}\right)$ is a sequence such that $x_{n} \nearrow x_{0}$. Let $\phi$ be the minimal solution of $D x(t) \geq v(x(t))$ for $t \in[0, t], x(0)=x_{0}$, then $\phi_{n} \rightarrow \phi$ uniformly on $[0, T]$.

Proof. By (2.7), it is enough to prove it for $D=D_{+}$. Consider the sequence $\left(\phi_{n}\right)$. If $i \leq j$ then $\phi_{i} \leq \phi_{j}$, otherwise there exists s, such that $\phi_{i}(s)>\phi_{j}(s)$. We write $\phi(t)=\left(\phi_{i} \wedge \phi_{j}\right)(t)$, obviously $D_{+} \phi(t)=D_{+} \phi_{i}(t)$ if $\phi_{i}(t)<\phi_{j}(t)$ and $D_{+} \phi(t)=\min \left\{D_{+} \phi_{i}(t), D_{+} \phi_{j}(t)\right\}$ if $\phi_{i}(t)=\phi_{j}(t)$. Then $\phi$ is a solution of $D_{+} x(t) \geq v(x(t))$, for $t \in[0, T], x(0)=x_{i}$. But $\phi(s)=\phi_{j}(s)<$ $\phi_{i}(s)$ so, this is contradiction to the minimality of $\phi_{i}$. Thus $\phi_{i}(t) \leq \phi_{j}(t)$ for all $t \in[0, T]$.

Clearly, $D_{+} \phi_{n}(t) \geq k_{1}$ for all $t \in[0, T]$, so by $(1.5), \phi_{n}(t) \geq k_{1} t+x_{n}$ for all $t \in[0, T]$. Then by the intermediate value theorem there exists $\varepsilon_{n}>0$ that $\phi_{n}\left(\varepsilon_{n}\right)=x_{0}=\phi(0)$, because $x_{n}<x_{0}$. Therefore $x_{0} \geq k_{1} \varepsilon_{n}+x_{n}$ so, $\left(x_{0}-x_{n}\right) / k_{1} \geq \varepsilon_{n}$ and by letting $n \rightarrow \infty, \varepsilon_{n} \rightarrow 0$. Since $\phi_{n}\left(\varepsilon_{n}\right)=x_{0}=\phi(0)$, we conclude that $\phi_{n}\left(t+\varepsilon_{n}\right)$ is a solution of $D_{+} x(t) \geq v(x(t)), x(0)=x_{0}$. Thus, by minimality of $\phi,(1) \phi_{n}\left(t+\varepsilon_{n}\right) \geq \phi(t)$ for all $t \in[0, T]$. We define now $\psi_{n}$ agrees with $\phi_{n}$ on $\left[0, \varepsilon_{n}\right]$ and agrees with $\phi\left(t-\varepsilon_{n}\right)$ for $t \geq \varepsilon_{n}$. Then obviously 
$D_{+} \psi_{n}(t) \geq v\left(\psi_{n}(t)\right), \psi_{n}(0)=x_{n}$. Hence $\psi_{n}(t)=\phi\left(t-\varepsilon_{n}\right) \geq \phi_{n}(t)$ for all $t \geq \varepsilon_{n}$, by minimality of $\phi_{n}$. We replace $t-\varepsilon_{n}$ by $t$ so we obtain $(2) \phi(t) \geq$ $\phi_{n}\left(t+\varepsilon_{n}\right)$ for all $t \geq 0$. Now, by (1) and (2) we have (3) $\phi(t)=\phi_{n}\left(t+\varepsilon_{n}\right)$ for all $t \in[0, T]$. Obviously $k_{1} \leq D_{+} \phi_{n}(t) \leq k_{2}$ for all $t \in[0, T]$, then so $\left(\phi_{n}\right)$ is equicontinuous. Then by $(3), \phi(t)=\lim _{n} \phi_{n}\left(t+\varepsilon_{n}\right)=\lim _{n} \phi_{n}(t)$. Hence $\left(\phi_{n}\right)$ is point wise convergent to $\phi$. Since $\left(\phi_{n}\right)$ is uniformly bounded, by the ArzelaAscoli theorem, $\left(\phi_{n}\right)$ has a subsequence which converges uniformly. But $\left(\phi_{n}\right)$ is monotone, therefore $\phi_{n} \rightarrow \phi$ uniformly.

5.5 LEMMA. Suppose that the hypotheses of the lemma (5.4) are satisfied but $x_{n} \searrow x_{0}$. Then as in (5.4), $\phi_{n} \rightarrow \phi$ uniformly on $[0, T]$.

Proof. $\quad$ Since $D \phi(t) \geq k_{1}$ for all $t \in[0, T]$, by (1.3), $\phi(t) \geq k_{1} t+$ $x_{0}$ for all $t \in[0, T]$. Then by the intermediate value theorem, for each $n$, $\phi\left(\varepsilon_{n}\right)=x_{n}=\phi_{n}(0)$ for some $\varepsilon_{n}>0$. Then $\phi\left(\varepsilon_{n}\right)=x_{n} \geq k_{1} \varepsilon_{n}+x_{0}$, and so $\varepsilon_{n} \leq\left(x_{n}-x_{0}\right) / k_{1}$ for all $n$. Hence $\varepsilon_{n} \rightarrow 0$ as $n \rightarrow \infty$. Clearly $\phi\left(t+\varepsilon_{n}\right)$ is a solution of $D x(t) \geq v(x(t)), t \in[0, T], x(0)=x_{n}$. So by minimality of $\phi_{n},(1) \phi\left(t+\varepsilon_{n}\right) \geq \phi_{n}(t)$ for all $t \in[0, T]$. We define now, $\psi_{n}(t)=\phi(t)$ on $\left[0, \varepsilon_{n}\right], \psi_{n}(t)=\phi_{n}\left(t-\varepsilon_{n}\right)$ for $t \in\left[\varepsilon_{n}, T\right]$. Obviously $\psi_{n}$ is a solution of $D x(t) \geq v(x(t)), t \in[0, T], x(0)=x_{0}$, thus $\psi_{n}(t)=\phi_{n}\left(t-\varepsilon_{n}\right) \geq \phi(t)$ for $t \in\left[\varepsilon_{n}, T\right]$, by minimality of $\phi$. We replace now, $t-\varepsilon_{n}$ by $\mathrm{t}$, so we have (2) $\phi_{n}(t) \geq \phi\left(t+\varepsilon_{n}\right)$ for all $t \in[0, T]$. By (1), (2) we conclude $\phi_{n}(t)=\phi\left(t+\varepsilon_{n}\right)$ for all $t \in[0, T]$, and all $n$. Hence $\left|\phi(t)-\phi_{n}(t)\right|=\left|\phi(t)-\phi\left(t+\varepsilon_{n}\right)\right|$ for all $t \in[0, T]$. Since $\varepsilon_{n} \rightarrow 0$ as $n \rightarrow \infty,\left|\phi(t)-\phi\left(t+\varepsilon_{n}\right)\right| \rightarrow 0$. So, $\phi_{n} \rightarrow \phi$ pointwise and uniformly as in the lemma (5.4).

5.6 COROLLARY. Let $u: R \rightarrow R$ be usc function with $k_{2} \geq u(x) \geq$ $k_{1}>0$ (or $\left.k_{2}<0\right)$. Assume $\phi_{n}$ is the maximal solution of $D x(t) \leq u(x(t))$ for $t \in[0, T], x_{0}=x_{n}$, where $\left(x_{n}\right)$ is a sequence such that $x_{n} \rightarrow x_{0}$. Also let $\phi$ be the maximal solution of $D x(t) \leq u(x(t))$, for $t \in[0, T], x(0)=x_{0}$. Then $\phi_{n} \rightarrow \phi$ uniformly on $[0, T]$.

Proof. We consider first, $x_{n} \nearrow x_{0}$ and second $x_{n} \searrow x_{0}$. Also we use us.continuity instead of ls.continuity and obviously reverse the inequalities.

5.7 THEOREM. Let $F(x)=[v(x), u(x)]$ be an usc interval-valued map with $0<k_{1} \leq F(x) \leq k_{2}$ (or $k_{2}<0$ ) for all $x$. Let $\phi_{n}$ be the maximal (resp. minimal) solution of $D x(t) \in F(x(t))$ for $t \in[0, T]$ and $x(0)=x_{n}$, where $\left(x_{n}\right)$ is a sequence such that $x_{n} \rightarrow x_{0}$. Also, suppose $\phi$ is the maximal (resp. minimal) solution of $D x(t) \in F(x(t)), x(0)=x_{0}$. Then $\phi_{n} \rightarrow \phi$ uniformly on $[0, T]$.

Proof. The proof by (5.4), (5.5) and (5.6) is obvious.

5.8 THEOREM. Let $u: R \rightarrow R$ be a usc function with $k_{2} \geq u(x) \geq k_{1}>0$ (or $\left.k_{2}<0\right)$. Assume $\phi(t, x)$ is the maximal solution of $D x(t) \leq u(x(t)), x(0)=$ 
$x$ for $t \in[0, T]$. Then $(t, x) \rightarrow \phi(t, x)$ is continuous.

Proof. $\quad$ By (5.6) for $t \in[0, T], \phi\left(t, x_{n}\right) \rightarrow \phi(t, x)$ uniformly as $x_{n} \rightarrow x$ and also $t \rightarrow \phi(t, x)$ is continuous for each $x \in R$, so $(t, x) \rightarrow \phi(t, x)$ is continuous. Because $[0, T]$ is compact and $R$ is metrizable. For more details see [3,p.314].

There are the same result for $l s c$ functions.

5.9 COROLLARY. Let $v: R \rightarrow R$ be an lsc function with $k_{2} \geq v(x) \geq$ $k_{1}>0$ (or $\left.k_{2}<0\right)$. Assume $\phi(t, x)$ is the minimal solution of $D x(t) \geq$ $v(x(t)), x(0)=x$ for all $t \in[0, T]$. Then $(t, x) \rightarrow \phi(t, x)$ is continuous.

Proof. The proof is the same as the proof of (5.8).

5.10 THEOREM. Let $F(x)=[v(x), u(x)]$ be as in (5.7). Suppose $\phi(t, x)$ be maximal (or minimal) solution of $D x(t) \in F(x(t)), x(0)=x$ for all $t \in[0, T]$. Then $(t, x) \rightarrow \phi(t, x)$ is continuous.

Proof. The proof by (5.7), (5.8) and (5.9) is clear.

In the preceding lemmas, the conditions $v(x) \geq k>0$ and $u(x) \geq k>0$ (or $v(x) \leq k<0$ and $u(x) \leq k<0$ ) are essential. To illustrate this, consider the usc function $u(x)$ as follows.

5.11 Example. $\quad$ Suppose usc set-valued map $u:[0,2] \rightarrow R$ is defined by:

$$
u(x)= \begin{cases}1 / 2^{n-1} & 1-1 / 2^{n-1}<x \leq 1-1 / 2^{n}, \\ 1 & 0 \leq x \leq 1 / 2 \text { or } 1 \leq x \leq 2,\end{cases}
$$

for all $n$. The solution for initial value $x_{0} \geq 1$ will be $\phi(t)=t+x_{0}$ and for $x_{0}<1$ the solution will not be a straight line (see figure 5). Thus if $x_{n} \nearrow x_{0}=1$ and $\phi_{n}$ is the solution for initial value $x_{n}$, then $\phi_{n}$ doesn't tend to $\phi$. The graphs of some solution curves are shown in the following figures.

\section{REFERENCES}

[1] T. M. Flett, Differential Analysis, Cambridge University Press, 1980.

[2] A. M. Forouzanfar, Compact right upper semi-continuous semigroups of closed sets, Semigroup Forum, 42 (1991), 175-188.

[3] J. L. Kelly, General Topology, Van Nostrand, 1955.

[4] K. Kuratowski, Topology, Academic Press, New York - London, 1968.

[5] V. Lakshmikantham, Upper and lower bounds of the norm of solutions of differential equations, Proc. Amer. Math. Soc. 13 (1962).

[6] E. Michael, Topologies on spaces of subsets, Trans. Amer. Math. Soc. 71 (1951), 152-182.

\section{Received: July 31, 2006}




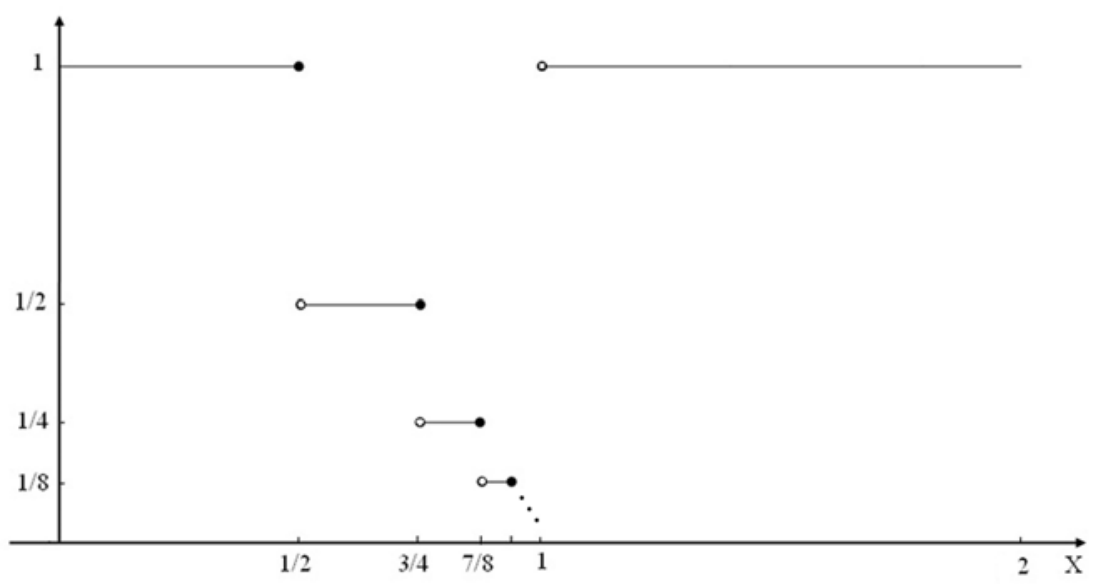

Figure 4: Graph of $u$.

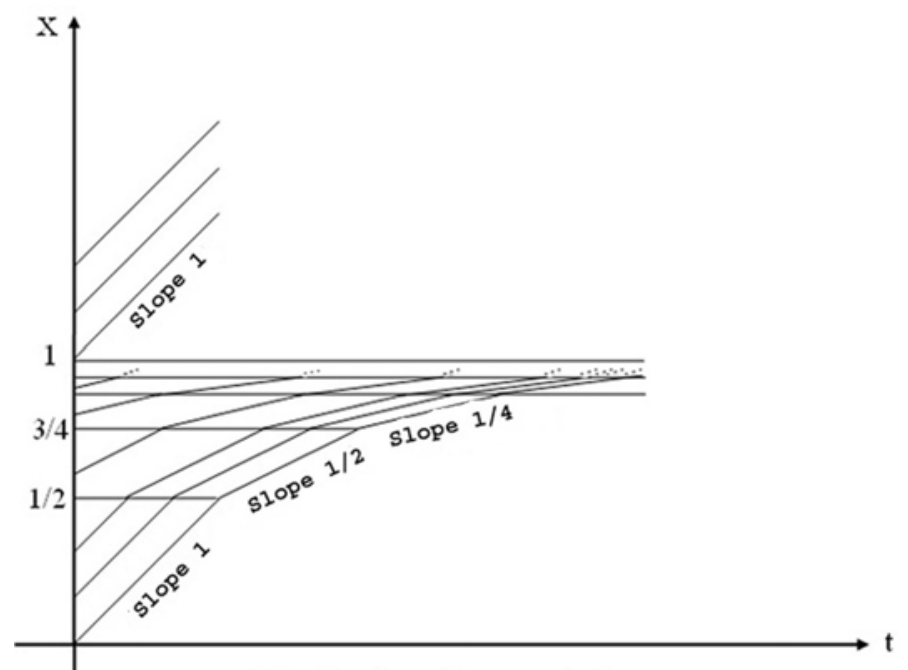

Figure 5: Graph of some solutions. 